\title{
Cyberknife Radiosurgery in Hepatocellular Carcinoma
}

\author{
Bakhtawar Memon, Shazia Kadri, Nimrah Sultana, Kamran Saeed, Naveed Ahmed and Tariq Mahmood \\ Department of Radiology and Cyberknife Robotic Radiosurgery, Jinnah Postgraduate Medical Centre, Karachi, Pakistan
}

\begin{abstract}
Objective: To determine the outcome of Stereotactic Body Radiation Therapy (SBRT) and its prognostic factors among hepatocellular carcinoma (HCC) patients.

Study Design: Descriptive study.

Place and Duration of Study: Department of Radiology and Cyberknife Robotic Radiosurgery, Jinnah Postgraduate Medical Centre (JPMC), Karachi, Pakistan from 1 1t July 2019 to $31^{\text {st }}$ August 2020.

Methodology: All patients of either gender age 18 years and above presenting with pathological confirmation of HCC, ECOG performance status of $\leq 2$ and child Pugh A or B were consecutively enrolled. Progression of $>20 \%$ was defined as progressive disease (PD). SBRT was performed using the Cyberknife. Clinical outcome was measured in terms of progression free survival. Moreover, radiation induced toxicity was also observed along with other predictor variables.

Results: Of 52 patients, the median age was 58 (53-59) years. There were $42(80.8 \%)$ males and $10(19.2 \%)$ females. All patients were alive at 3 months, i.e. $52(100 \%)$. However, at 6 months, $49(94.2 \%)$ were alive, at 9 months, 38 (73.1\%) patients were alive while at 1 year, $24(46.2 \%)$ patients were alive. A significant association was observed for survival at 6 months and ECOG performance status score ( $p$-value 0.036), survival at 9 months and AFP $(p=0.003)$, survival at 1 year and age $(p=$ $0.019)$, survival at 1 year and HBV $(p=0.001)$, and survival at 1 year and previous treatment $(p=0.010)$. Moreover, none of the patients reported complications / radiation induced liver toxicity.
\end{abstract}

Conclusion: A higher efficiency of SBRT was found among HCC patients attending Radiology Department.

Key Words: Stereotactic body radiation therapy, Hepatocellular carcinoma, Toxicity, Survival.

How to cite this article: Memon B, Kadri S, Sultana N, Saeed K, Ahmed N, Mahmood T. Cyberknife Radiosurgery in Hepatocellular Carcinoma. J Coll Physicians Surg Pak 2021; 31(05):532-536.

\section{INTRODUCTION}

Hepatocellular carcinoma $(\mathrm{HCC})$ is the third leading cause of cancer-related death worldwide. ${ }^{1,2}$ Moreover, it is one of the most common solid tumors highly prevalent in all over the world. ${ }^{2}$ It is reported that number of cases of HCC are continuously increasing in Pakistan. ${ }^{3}$ It is now, one of the commonest cancers in adult males in the country. Estimates show that 2.8 per 100,000 females and 7.6 per 100,000 males are affected by HCC in Pakistan. ${ }^{4}$ The accurate and timely diagnosis of HCC has thus become an utmost requirement to halt the uprising trend of HCC.

Various treatment options are available for HCC, such as surgery, percutaneous radio-frequency ablation and chemoembolisation. ${ }^{5,6}$

Correspondence to: Dr. Bakhtawar Memon, Department of Radiology and Cyberknife Robotic Radiosurgery, Jinnah Postgraduate Medical Centre, Karachi, Pakistan

E-mail: bakhtawar2004@hotmail.com

Received: November 12, 2020; Revised: March 18, 2021;

Accepted: April 09, 2021

DOI: https://doi.org/10.29271/jcpsp.2021.05.532
In addition to all these management strategies, radiation therapy is also now being conducted for the treatment of HCC. Stereotactic body radiation therapy (SBRT) is the treatment modality, which is now currently being practised world wide. It is reported that SBRT is a recommended treatment option for HCC patients, who are ineligible for previously recommended treatment modalities. ${ }^{7,8}$ Various international studies have reported good clinical outcome of SBRT ${ }^{7-9}$ However, an extensive literature search has revealed scarcity of data from Pakistan on the outcome of SBRT in patients with HCC.

Therefore, this study was planned with the aim to determine the outcome of SBRT and its prognostic factors among HCC patients attending Radiology Department of a large public sector hospital in Karachi, Pakistan.

\section{METHODOLOGY}

This descriptive cohort study was prospectively conducted at Department of Radiology and Cyberknife Robotic Radiosurgery, Jinnah Postgraduate Medical Centre, Karachi from July 2019 to August 2020.

All patients of either gender, age 18 years and above presenting with pathological confirmation of HCC, ECOG performance status of $\leq 2$ and child Pugh A or B, were consecutively enrolled. While all patients with extrahepatic extensions, previous radio- 
therapy for liver tumors, child-pugh $\mathrm{C}$, intractable ascites and normal liver volume of less than 700 cc were excluded. Imaging criteria for HCC included arterial phase hyper-enhancing lesion, with showing washout on porto-venous and delayed phase graded and stage III-B (T4,NO.MO) without direct invasion of adjacent organs or perforation. Epi Info sample size calculator was used for the estimation of sample size. Taking confidence interval 95\%, reported complete response in a previous study $87.5 \%{ }^{10}$, margin of error $9 \%$, the sample size came out to be 52 .

Clinical outcome was measured in terms of progression-free survival. Progression of $>20 \%$ was defined as progressive disease (PD). Progression-free survival was defined as the number of patients remain alive during the one-year follow-up. Risk factors like chronic hepatitis $B$ and $C$, hepatitis $B$ carrier state, prior transcatheter arterial embolization, concurrent chemotherapy, portal vein thrombosis, high tumor staging and male gender, were observed.

Relevant investigations were done at the time of patient's registration, including liver triphasic computed tomography (CT), complete blood picture, liver function tests, PCR virus testing for hepatitis B and C, serum alpha-fetoprotein (AFP) and CT chest with contrast for any pulmonary metastasis. Patients with positive surface antigen for hepatitis B (HBsAg) or elevated hepatitis $B$ virus DNA were referred to gastroenterologist for treatment before SBRT. SBRT was performed using the cyberknife, an image-guided robotic radiosurgery system synchronous with the respiratory tracking for target lesions that move with respiration. Approximately 5-7 days before the planning CT-scan, under ultrasound guidance gold fiducial markers were implanted percutaneously around the target lesion. A CT scan with intravenous contrast having around 1-mm thin slice thickness, was performed covering the whole liver and bilateral kidneys. No abdominal compression or respiratory control was used at the time of scan. Contouring was done on cyberknife system's treatment planning workstation on the CT scan images transferred to it. (Figure 1). Beam directions and beam weights were automatically decided by the system, optimally in order to maximize the delivered dose to the target lesion and minimize radiation dose to the adjacent organs at risk.

Dosing was determined depending on size and location of tumor, normal liver volume and adjacent organs at radiation risk. Doses delivered to tumors ranged from 26 to 50 Gy in 3-5 fractions. The gross tumor volume (GTV) included tumor seen on CT scans with contrast. Marginal $5 \mathrm{~mm}$ was added as CTV (clinical target tumor) to cover microscopic disease. The SBRT radiation to the target lesion was delivered along with the real-time tracking system under fiducial markers guidance, synchronizing with the tracking system. The limited radiation dose for normal liver parenchyma (total liver minus cumulative GTV) was specified so that a liver volume of minimum $700 \mathrm{ml}$ could receive a total radiation dose less than $15 \mathrm{~Gy}$. The dose given to critical organs such as stomach, duodenum, colon, right kidney and spinal cord was in recommended ranges as specified by AAPM-TG 101. Follow-up was done at $3^{\text {rd }}$ month, $6^{\text {th }}$ month, $9^{\text {th }}$ month, and 1 year post SBRT therapy.

Statistical analysis was performed using SPSS version 24. Median and interquartile range was reported for quantitative variables like age of the patients and laboratory parameters. Frequency and percentages were calculated for qualitative variables like gender, HBV, HCV status, previous treatment history, child pugh score, and survival. Inferential statistics were explored using Friedman test, Fisher's Exact test/Chi-square test. p-value $\leq 0.05$ was considered as significant.

\section{RESULTS}

A total of 52 patients were included. The median age of the patients was 58 (53-59) years. There were $42(80.8 \%)$ males and 10 (19.2\%) females. Positive PCR for hepatitis B virus was found in 10 (19.2\%), while hepatitis C virus in 28 (53.8\%) patients; whereas, other had no obvious etiology. Solitary tumor was observed in 44 (84.6\%) patients, while 8 (15.4\%) patients had $\geq 2$ lesions in each group. Higher number of patients were found to have tumor in right lobe, i.e. 41 (78.8\%), followed by left side 7 (13.5\%) and both lobes were involved in $4(7.7 \%)$ patients. Thirteen $(25 \%)$ with unsuccessful previous treatment (TACE/RFA) were referred for cyberknife.

All patients were alive at 3 months, i.e., 52 (100\%). However, at 6 months, 49 (94.2\%) were alive, at 9 months, 38 (73.1\%) patients were alive, while at one year, 24 (46.2\%) patients were alive. The progression-free survival was observed in all of the alive patients. The cause of death was due to other factors, which includes development of new hepatic lesions, development of peritoneal ascites and extrahepatic metastasis.

Out of 52 patients at baseline and 3 months of follow-up, ECOG performance status grade 0 was found in $4(7.7 \%)$ and $2(3.8 \%)$ patients, respectively. Of 49 patients at 6 months, ECOG performance status grade 0 was found in $3(6.1 \%)$, while of 24 patients at one year, ECOG performance status grade 0 was found in 2 $(8.3 \%)$ patients.

At baseline, child pugh score A was found in 51 (98.1\%) patients. Of these 51 patients, 42 (82.4\%) remains same at $3^{\text {rd }}$ month. The follow-up of these patients showed that at 6 and 9 months, child pugh score remained the same, i.e. 29/48 (60.4\%) and $23 / 38(60.5 \%)$, respectively. (Figure 2) Finally, at one year, among 24 of those who survived, 19 (79.2\%) still had child pugh score A. (Figure 3) One patient whose child score was B at the time of enrollment, survived till 9 months with the same status. However, the patient expired at 1-year follow-up due to development of newlesion.

A significant association was observed between survival at 6 months and ECOG performance status score $(p=0.036)$, survival at 9 months and AFP ( $p=0.003)$, survival at one year and age $(p=0.019)$, survival at one year and HBV $(p=0.001)$, and survival at one year and previous treatment $(p=0.010$, Tablel). 
Table I: Comparative analysis of survival at different time intervals with predictor variables $(n=52)$.

\begin{tabular}{|c|c|c|c|c|c|c|c|c|c|}
\hline & \multicolumn{3}{|c|}{ Survival at 6 months } & \multicolumn{3}{|c|}{ Survival at 9 months } & \multicolumn{3}{|c|}{ Survival at 1 year } \\
\hline & Yes $(n=49)$ & No $(n=3)$ & p-values & Yes $(n=38)$ & No $(n=14)$ & p-values & Yes $(n=24)$ & No $(n=28)$ & p-values \\
\hline \multicolumn{10}{|l|}{ Age, n (\%) } \\
\hline$\leq 58(n=30)$ & $30(100)$ & $0(0)$ & \multirow{2}{*}{$0.07^{\neq}$} & $25(83.3)$ & $5(16.7)$ & \multirow{2}{*}{$0.052^{\wedge}$} & $18(60.0)$ & $12(40.0)$ & \multirow{2}{*}{$0.019^{\wedge}$} \\
\hline$>58(n=12)$ & $19(86.4)$ & $3(13.6)$ & & $13(59.1)$ & $9(40.9)$ & & $6(27.3)$ & $16(72.7)$ & \\
\hline \multicolumn{10}{|l|}{ Gender, n (\%) } \\
\hline Male $(n=42)$ & $39(92.9)$ & $3(7.1)$ & \multirow{2}{*}{$>0.999^{\neq}$} & $30(71.4)$ & $12(28.6)$ & \multirow{2}{*}{$0.710^{\neq}$} & $21(50)$ & $21(50)$ & \multirow{2}{*}{$0.309^{\neq}$} \\
\hline Female $(n=10)$ & $10(100)$ & $0(0)$ & & $8(80.0)$ & $2(20.0)$ & & $3(30)$ & $7(70)$ & \\
\hline \multicolumn{10}{|l|}{ HBV, n (\%) } \\
\hline Yes $(n=42)$ & $41(97.6)$ & $1(2.4)$ & \multirow{2}{*}{$0.091^{\neq}$} & $33(78.6)$ & $9(21.4)$ & \multirow{2}{*}{$0.109^{\neq}$} & $24(57.1)$ & $18(42.9)$ & \multirow{2}{*}{$0.001^{\neq}$} \\
\hline No $(n=10)$ & $8(80.0)$ & $2(20.0)$ & & $5(50)$ & $5(50)$ & & $0(0)$ & $10(100)$ & \\
\hline \multicolumn{10}{|l|}{$\mathrm{HCV}, \mathrm{n}(\%)$} \\
\hline Yes $(n=24)$ & $22(91.7)$ & $2(8.3)$ & \multirow{2}{*}{$0.590^{\neq}$} & $17(70.8)$ & $7(29.2)$ & \multirow{2}{*}{$0.736^{\wedge}$} & $8(33.3)$ & $16(66.7)$ & \multirow{2}{*}{$0.086^{\wedge}$} \\
\hline No $(n=28)$ & $27(96.4)$ & $1(3.6)$ & & $21(75.0)$ & $7(25.0)$ & & $16(57.1)$ & $12(42.9)$ & \\
\hline \multicolumn{10}{|c|}{ Previous Treatment, $\mathrm{n}(\%)$} \\
\hline Yes $(n=13)$ & $13(100)$ & $0(0)$ & \multirow{2}{*}{$0.564^{\neq}$} & $11(84.6)$ & $2(15.4)$ & \multirow{2}{*}{$0.472^{\neq}$} & $10(76.9)$ & $3(23.1)$ & \multirow{2}{*}{$0.010^{\wedge}$} \\
\hline No $(n=39)$ & $36(92.3)$ & $3(7.7)$ & & $27(69.2)$ & $12(30.8)$ & & $14(35.9)$ & $25(64.1)$ & \\
\hline \multicolumn{10}{|l|}{ ECOG, $\mathrm{n}(\%)$} \\
\hline $0(n=4)$ & $3(75.0)$ & $1(25.0)$ & \multirow{3}{*}{$0.036^{\wedge}$} & $3(75.0)$ & $1(25.0)$ & \multirow{3}{*}{$0.985^{\wedge}$} & $2(50)$ & $2(50)$ & \\
\hline $1(n=34)$ & $34(100)$ & $0(0)$ & & $25(73.5)$ & $9(26.5)$ & & $14(41.2)$ & $20(58.8)$ & $0.594^{\wedge}$ \\
\hline $2(n=14)$ & $12(85.7)$ & $2(14.3)$ & & $10(71.4)$ & $4(28.6)$ & & $8(57.1)$ & $6(42.9)$ & \\
\hline Child Pugh Scor & $\mathrm{n}(\%)$ & & & & & & & & \\
\hline$A(n=51)$ & $48(94.1)$ & $3(5.9)$ & & $37(72.5)$ & $14(27.5)$ & & $24(47.1)$ & $27(52.9)$ & \\
\hline$B(n=1)$ & $1(100)$ & $0(0)$ & $>0.999^{-}$ & $1(100)$ & $0(0)$ & $>0.999^{-}$ & $0(0)$ & $1(100)$ & $>0.999^{-}$ \\
\hline $\mathrm{ALT}, \mathrm{n}(\%)$ & & & & & & & & & \\
\hline$\leq 30(n=32)$ & $30(93.8)$ & $2(6.3)$ & & $25(78.1)$ & $7(21.9)$ & & $17(53.1)$ & $15(46.9)$ & \\
\hline$>30(n=20)$ & $19(95.0)$ & $1(5.0)$ & $>0.999^{7}$ & $13(65.0)$ & $7(35.0)$ & $0.299^{\wedge}$ & $7(35.0)$ & $13(65.0)$ & $0.202^{\wedge}$ \\
\hline ALP, n (\%) & & & & & & & & & \\
\hline$\leq 70(n=30)$ & $27(90.0)$ & $3(10.0)$ & & $21(70.0)$ & $9(30.0)$ & & $11(36.7)$ & $19(63.3)$ & \\
\hline$>70(n=22)$ & $22(100)$ & $0(0)$ & $0.253^{7}$ & $17(77.3)$ & $5(22.7)$ & $0.559^{\wedge}$ & $13(59.1)$ & $9(40.9)$ & $0.109^{\wedge}$ \\
\hline AFP, n (\%) & & & & & & & & & \\
\hline$\leq 18(n=25)$ & $24(96.0)$ & $1(4.0)$ & & $23(92.0)$ & $2(8.0)$ & & $12(48.0)$ & $13(52.0)$ & \\
\hline$>18(n=27)$ & $25(92.6)$ & $2(7.4)$ & $>0.999^{7}$ & $15(55.6)$ & $12(44.4)$ & $0.003^{\wedge}$ & $12(44.4)$ & $15(55.6)$ & $0.797^{\wedge}$ \\
\hline Platelets, n (\%) & & & & & & & & & \\
\hline$\leq 18(n=26)$ & $24(92.3)$ & $2(7.7)$ & & $18(69.2)$ & $8(30.8)$ & & $12(46.2)$ & $14(53.8)$ & \\
\hline$>18(n=26)$ & $25(96.2)$ & $1(3.8)$ & $>0.999^{-}$ & $20(76.9)$ & $6(23.1)$ & $0.532^{\wedge}$ & $12(46.2)$ & $14(53.8)$ & $>0.999^{\wedge}$ \\
\hline
\end{tabular}

Table II: Median platelet count at different time interval.

\begin{tabular}{|c|c|c|}
\hline \multicolumn{3}{|c|}{ Platelets Count } \\
\hline \multicolumn{3}{|c|}{ Overall $(n=52)$} \\
\hline Baseline & $151.5(125.0-179.5)$ & \multirow{5}{*}{0.339} \\
\hline At 3 months & $150(114.0-181.5)$ & \\
\hline At 6 months & $147(100-156.5)$ & \\
\hline At 9 months & $147(110-157)$ & \\
\hline At 1 year & $147.5(119-187.5)$ & \\
\hline \multicolumn{3}{|c|}{ With previous treatment $(n=13)$} \\
\hline Baseline & $168(129.5-196)$ & \multirow{5}{*}{0.092} \\
\hline At 3 months & $150(130-188)$ & \\
\hline At 6 months & $147(134.5-150)$ & \\
\hline At 9 months & $145(125-156)$ & \\
\hline At 1 year & $145(117.5-182.3)$ & \\
\hline \multicolumn{3}{|c|}{ No previous treatment $(n=39)$} \\
\hline Baseline & $150(116-178)$ & \multirow{5}{*}{0.275} \\
\hline At 3 months & $141.5(100-185.3)$ & \\
\hline At 6 months & $146(100-159.5)$ & \\
\hline At 9 months & $150(109-160)$ & \\
\hline At 1 year & $150(116.8-190)$ & \\
\hline
\end{tabular}

An insignificant median difference was observed for platelet counts at different time intervals $(p=0.339)$. The median platelet count at baseline was 151.5 (125-179.5), at 3 months was 150.0 (114-181.5), at 6 months was 147 (100-156.5), at 9 months was 147.0 (110-157), while at 12 months was 147.5 (119-187.5, Table II). Furthermore, ALT ( $p$ $=0.217$ ) and ALP levels ( $p=0.208$ ) were also found to be insignificant at different time intervals.

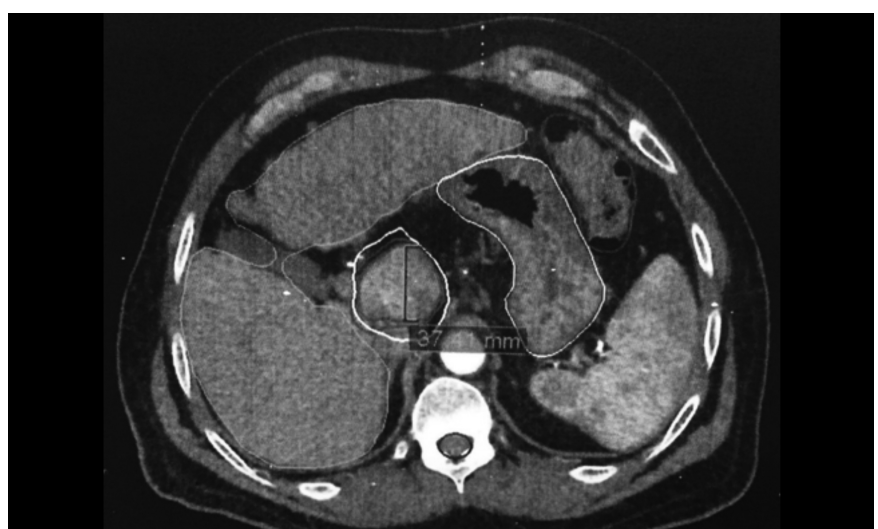

Figure 1: Contouring performed on planning CT images with contrast on Cyberknife system's treatment planning workstation 


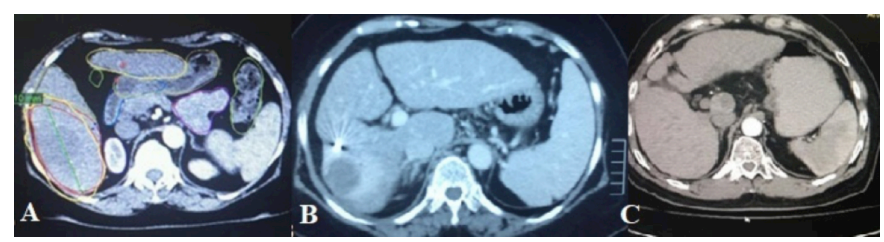

Figure 2: A 60 years patient showing arterially enhancing lesion in segment $\mathrm{VI}$ of liver (A), which shows central necrotic area with peripheral edema on 3 months follow Up (B) representing SBRT changes. On 1 year follow up, complete regression of lesion is seen, representing therapy response $(C)$. Note the hyperdense fudicial in image $B$.

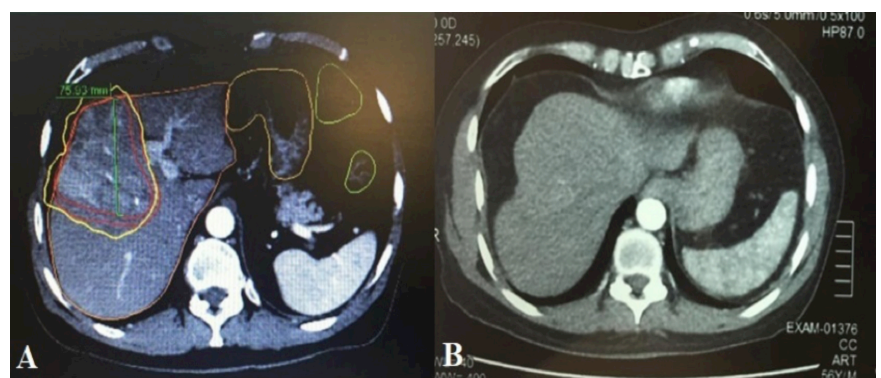

Figure 3: A 56 years patient showing arterially enhancing lesion in Segment VIII of liver (A), which appears to be less enhancing on 6 months follow up (B), representing response to therapy.

\section{DISCUSSION}

Currently, SBRT is one of the most recommended therapies worldwide. ${ }^{8-10}$ Similarly, in Pakistan, this therapy is also used for the management of HCC. SBRT is newly emerging treatment option for management of various carcinomas including hepatocellular carcinoma. The main advantage of SBRT is to maximize the radiation dose to the target lesion to control the local spread of disease, minimizing the dose to adjacent organs at risk. ${ }^{11-13}$ Scarcity of the data from Pakistan on SBRT has urged us to report local outcome of SBRT. The findings of the current study have reported favorable outcome of SBRT with no significant complications. Though, an extensive literature has revealed dearth of studies from Pakistan that has reported cyberknife surgery, except for a similar kind of study presented by Kadri et al. in European Congress of Radiology (ECR) in 2017 from Karachi, in which outcome of eight patients of HCC was reported, using cyberknife SBRT treatment. ${ }^{14}$ The findings of that study revealed that among eight patients, tumor size and serum AFP were reduced in four lesions, tumor size remained stable in three lesions and disease progression noted in one lesion. Moreover, complication was observed in none of the patients in the study by Kadri et al. ${ }^{14}$ The author concluded that SBRT was rising as a new, promising, non-invasive therapy with minimal or no side effects for patients with unresectable hepatocellular carcinoma. Small tumors showing good results, though few larger tumors have been successfully treated as well.

In the current study, all 52 patients were alive at the third month. However, at sixth month, almost $94 \%$ percent patients were alive; at ninth month, $73 \%$ patients were alive, while at one year, survival status reached $46 \%$. The progres- sion-free survival was observed in all of the alive patients. The cause of death was due to other factors, which includes development of new hepatic lesions, and peritoneal ascites or extrahepatic metastasis. Kwon et al. in their study enrolled HCC patients not eligible for standard treatment and have assessed long term effects of SBRT for primarily small HCC. ${ }^{15}$ The outcome of their study has revealed overall one- and three-year survival rates of approximately $93 \%$ and $59 \%$, respectively. The local control rates at one- and three-year were $72 \%$ and $68 \%$, respectively. The author finally stated that smaller tumors had better local control and overall survival rates. ${ }^{15}$ Sanuki et al. also evaluated small HCC patients treated with SBRT. ${ }^{16}$ The three years local control and overall survival rates in the $35 \mathrm{~Gy}$ and $40 \mathrm{~Gy}$ groups were $91 \%$ and $89 \%$, respectively. The author stated that similar outcome was observed in either dose in five fractions. Whereas, safety was also observed in all cases. ${ }^{16}$

Central liver toxicity after SBRT treatment and radiation-induced liver toxicity were reported in various previous studies. ${ }^{17-21}$ Likewise, central liver toxicity was also observed in the current study and no toxicity was observed.

The limitations of this study are that certain important variables like metabolic liver function are not reported in the current study. Moreover, the follow-up time was only one year in the current study. Despite these limitations, there are several strengths of this study. Firstly, this study is the first kind of study that has reported relatively ample number of HCC cases, who received SBRT therapy. Moreover, none of the patients has reported radiation-induced complication$\mathrm{s}$ /radiation-induced liver toxicity in the current study, which states SBRT as highly recommended therapy for patients with HCC, especially for those with failed prior treatment options. Finally, the current study has reported local data of SBRT of HCC patients from public sector hospital of Pakistan that offers free- of-cost robotic surgery.

\section{CONCLUSION}

There is high efficiency of SBRT among HCC patients without any major toxicity.

\section{ETHICAL APPROVAL:}

Ethical approval was obtained from JPMC prior to conducting the study.

\section{PATIENTS' CONSENT:}

Signed consents were taken from all eligible study participants.

\section{CONFLICT OF INTEREST:}

The authors declared no conflict of interest.

\section{AUTHORS' CONTRIBUTION:}

BM: Patient registration, data collection, analysis, article writing, follow-up assessment.

SK: Follow-up of patients on $3^{\text {rd }}, 6^{\text {th }}, 9^{\text {th }}$ and 1 year. 
NS: Data collection.

KS: Patient's assessment, planning SBRT treatment.

NA: Ultrasound-guided fudicial placement in every patient pre-treatment (SBRT).

TM: Head of department, supervisior.

\section{REFERENCES}

1. Ozakyol A. Global epidemiology of hepatocellular carcinoma (HCC epidemiology). J Gastrointest Cancer 2017; 48(3):238-40. doi: 10.1007/s12029-017-9959-0.

2. Sayiner M, Golabi P, Younossi ZM. Disease burden of hepatocellular carcinoma: A global perspective. Dig Dis Sci 2019; 64(4):910-7. doi: 10.1007/s10620-019-05537-2

3. Abbas Z. Hepatocellular carcinoma in Pakistan. J Coll Physicians Surg Pak 2013; 23(11):769-70.

4. Hafeez Bhatti AB, Dar FS, Waheed A, Shafique K, Sultan F, Shah NH. Hepatocellular carcinoma in Pakistan: National trends and global perspective. Gastroenterol Res Pract 2016; 2016. doi: 10.1155/2016/5942306.

5. Dhir M, Melin AA, Douaiher J, Lin C, Zhen WK, Hussain SM, et al. A review and update of treatment options and controversies in the management of hepatocellular carcinoma. Ann Surg 2016; 263(6):1112-25. doi: 10.1097/SLA.00000000 00001556.

6. Xie ZB, Ma L, Wang XB, Bai T, Ye JZ, Zhong JH, et al. Transarterial embolisation with or without chemotherapy for advanced hepatocellular carcinoma: A systematic review. Tumor Biol 2014; 35(9):8451-9. doi: 10.1007/s13277014-2340-z.

7. Murray LJ, Dawson LA. Advances in stereotactic body radiation therapy for hepatocellular carcinoma. Semin Radiat Oncol 2017; 27(3):pp. 247-255). doi: 10.1016/j.semradonc.2017.02.002.

8. Sapir E, Tao Y, Schipper MJ, Bazzi L, Novelli PM, Devlin P, et al. Stereotactic body radiation therapy as an alternative to transarterial chemoembolisation for hepatocellular carcinoma. Int J Radiat Oncol Biol Phys 2018; 100(1):122-30. doi: 10.1016/j.jjrobp.2017.09.001.

9. Velec M, Haddad CR, Craig T, Wang L, Lindsay P, Brierley J, et al. Predictors of liver toxicity following stereotactic body radiation therapy for hepatocellular carcinoma. Int J Radiat Oncol Biol Phys 2017; 97(5):939-46. doi: 10.1016/j.ijrobp.2017.01.221

10. Que J, Kuo HT, Lin LC, Lin KL, Lin CH, Lin YW, et al. Clinical outcomes and prognostic factors of cyberknife stereotactic body radiation therapy for unresectable hepatocellular carcinoma. BMC Cancer 2016; 16(1):451. doi: 10.1186/s12885016-2512-x.

11. Riou O, Moscardo CL, Fenoglietto P, Deshayes E, Tetreau R, Molinier J, et al. SBRT planning for liver metastases: A focus on immobilization, motion management and planning imaging techniques. Rep Pract Oncol Radiother 2017; 22(2):103-10. doi: 10.1016/j.rpor.2017.02.006.

12. Kellock T, Liang T, Harris A, Schellenberg D, Ma R, Ho S, et al. Stereotactic body radiation therapy (SBRT) for hepatocellular carcinoma: imaging evaluation post treatment. $\mathrm{Br} J$ Radiol 2018; 91(1085):20170118. doi: 10.1259/bjr.2017 0118.

13. Høyer M, Swaminath A, Bydder S, Lock M, Romero AM, Kavanagh $B$, et al. Radiotherapy for liver metastases: A review of evidence. Int J Radiat Oncol Biol Phys 2012; 82(3):1047-57. doi: 10.1016/j.ijrobp.2011.07.020.

14. Kadri S, Ahmed N, Arooj S, Saeed K, Mahmood T. Cyberknife Robotic Radiosurgery in Hepatocellular carcinoma-An Initial experience. Eur Radiol-ECR 2017. dx.doi.org/10.1594/ecr2017/C-1690.

15. Kwon JH, Bae SH, Kim JY, Choi BO, Jang HS, Jang JW, et al. Long-term effect of stereotactic body radiation therapy for primary hepatocellular carcinoma ineligible for local ablation therapy or surgical resection. Stereotactic radiotherapy for liver cancer. BMC cancer 2010; 10(1):475. doi: 10.1186/1471-2407-10-475.

16. Sanuki N, Takeda A, Oku Y, Mizuno T, Aoki Y, Eriguchi T, et al. Stereotactic body radiotherapy for small hepatocellular carcinoma: A retrospective outcome analysis in 185 patients. Acta oncologica 2014; 53(3):399-404. doi: 10.3109/0284186X.2013.820342.

17. Toesca DA, Osmundson EC, von Eyben R, Shaffer JL, Lu P, Koong AC, et al. Central liver toxicity after SBRT: An expanded analysis and predictive nomogram. Radiother Oncol 2017; 122(1):130-6. doi: 10.1016/ j.radonc.2016.10.024.

18. Osmundson EC, Wu Y, Luxton G, Bazan JG, Koong AC, Chang DT. Predictors of toxicity associated with stereotactic body radiation therapy to the central hepatobiliary tract. Int J Radiat Oncol Biol Phys 2015; 91(5):986-94. doi: 10.1016/j.ijrobp.2014.11.028.

19. Jun BG, Kim YD, Cheon GJ, Kim ES, Jwa E, Kim SG, et al. Clinical significance of radiation-induced liver disease after stereotactic body radiation therapy for hepatocellular carcinoma. Korean J Intern Med 2018; 33(6):1093. doi: 10.3904/kjim.2016.412.

20. O'Connor JK, Trotter J, Davis GL, Dempster J, Klintmalm GB, Goldstein RM. Long-term outcomes of stereotactic body radiation therapy in the treatment of hepatocellular cancer as a bridge to transplantation. Liver Transpl 2012; 18(8):949-54. doi: 10.1002/lt.23439.

21. Dreher C, Høyer K, Fode MM, Habermehl D, Combs SE, Høyer M. Metabolic liver function after stereotactic body radiation therapy for hepatocellular carcinoma. Acta Oncologica 2016; 55(7):886-91. doi: 10.3109/0284186X. 2015.1137352. 